\title{
Physical and chemical characteristics of unground palm oil fuel ash cement mortars with nanosilica
}

\begin{abstract}
The present study focused on enhancing properties of unground palm oil fuel ash (UPOFA) mortars by incorporation of nanosilica (NS). To do so, 0.5-1.5\% NS was admixed in each series of 10-30\% UPOFA, respectively. The results revealed that incorporating NS offset the adverse effect of UPOFA on flowability, compressive strength and water absorption of mortars. Addition of NS increased the replacement level of UPOFA up to $20 \%$ with comparable compressive strength to control sample. SEM and XRD results showed that admixing NS led to improvement in the microstructure, and pozzolanic behavior of UPOFA mixtures at 28 days.
\end{abstract}

Keyword: Unground palm oil fuel ash; Nanosilica; Mechanical properties; Chemical composition; Microstructure; Mortars 